\title{
Management of congenital talipes equino varus using Ponseti method: 3 year follow up in 166 club feet
}

\author{
Rahul Bansal*, Angad Jolly, P. B. Mohammed Farook, Idris Kamran, \\ Syed Wahaj, Vikas Hanasoge
}

Department of Orthopedics Mysore Medical College \& Research Institute, Mysore, Karnataka, India

Received: 23 May 2017

Revised: 10 June 2017

Accepted: 12 June 2017

*Correspondence:

Dr. Rahul Bansal,

E-mail: 85rahulbansal@gmail.com

Copyright: () the author(s), publisher and licensee Medip Academy. This is an open-access article distributed under the terms of the Creative Commons Attribution Non-Commercial License, which permits unrestricted non-commercial use, distribution, and reproduction in any medium, provided the original work is properly cited.

\section{ABSTRACT}

Background: Our aim was to study the effectiveness of Ponseti method using Pirani score in children with club foot treated over the past three years at our hospital.

Methods: We studied 111 children with 166 idiopathic club feet who were treated at our teaching hospital between period of January 2012 and January 2017.The foot deformities were assessed using Pirani score at the time of first visit and the scores were recorded with each subsequent visit, with each casting and manipulation until correction of deformity. Tenotomy was performed on all the idiopathic club feet and continued with Steenbeek foot abduction brace $(\mathrm{FAB})$ and the scores were recorded with every follow up and the progress was noted. All the relevant data in terms of treatment and demographics were recorded with dates and maintained.

Results: Total of 166 feet of 111 children was treated out of which 72 were males and 39 were females. 55 children had bilateral involvement remaining were unilateral. Mean Pirani score was 5.5 (range 4-6) when the treatment was started. On an average 5.7 casts (range 3-9) were required before preforming a tenotomy. Tenotomy was performed on all the feet $(100 \%)$ with idiopathic club foot. Foot abduction orthosis was given to all the patients and 108 patients (97.3\%) were compliant. Mean Pirani score after three years of treatment was 0.26. Skin complications like blister formation were seen in three children during the course of the treatment. Four patients did not follow up and defaulted. Three patients had relapse or worsening of Pirani score. The recurrence or worsening of scores is thought to be due to poor compliance while using the foot abduction brace.

Conclusions: Ponseti method of treatment for CTEV is very effective, simple, non-invasive and convenient with excellent outcomes over long term with no significant complications.

Keywords: CTEV, Club foot, Ponseti cast, Pirani score, Foot deformity

\section{INTRODUCTION}

Congenital talipes equinovarus (CTEV) is also known as club foot it is the most common and complex congenital deformity that is difficult to correct. ${ }^{1}$ The incidence of CTEV is one in 1000 live births with male to female ratio being 3:1 and $40 \%$ of cases are bilateral. ${ }^{2}$ CTEV has four components midfoot cavus, ankle equinus, forefoot adductus and hindfood varus which involves medial rotation of calcaneum, navicular and cuboid bones with respect to talus, also they are adducted and inverted causing the deformities. ${ }^{2}$ Front of the foot is pronated with respect to hind foot even though the whole foot itself is supinated which gives rise to the cavus deformity. Most cases are idiopathic and occur in normal infants but it is also known to be associated with neuromuscular 
diseases like spina bifida, various syndromes like arthrogryposis or amniotic band syndrome these form secondary club foot. Postural clubfoot is purely due to position of neonate in utero. Clubfoot can be detected in utero using sonographic assessment but it is mainly a clinical diagnosis and radiographs can help in further assessment. The aim of treatment should is to achieve painless, pliable, plantigrade foot with correction of all the deformities which is functionally and cosmetically acceptable. Untreated cases can cause physical, psychological and soci-economic burden on patients as well as their families. Neglected CTEV is one of the most significant cause of physical disability among congenital musculoskeletal disorders. ${ }^{3}$ Management of club foot can be operative or non-operative but latter is accepted worldwide as an initial line of management for idiopathic CTEV. ${ }^{4}$ Combination of initial casting, extensive soft tissue release on the posterior medial side and bony procedures followed by casting again was the traditional method of treatment. ${ }^{5}$ These methods have a long term success rate of only $15 \%$ to $50 \% .^{6,7}$ A non-operative approach introduced by Ignacio Ponseti uses serial casts to correct the deformity followed by application of food abduction splint, percutaneous tenotomy may or may not be required. ${ }^{2}$ Ponseti's method has shown to have success rates of more than $90 \%$ over long term follow-up with advantage being that it is less invasive, painless with very low complication rates. ${ }^{8}$ This method has been successfully used to achieve good results when performed by trained clinical specialists in either a teaching hospital setting or in a developing country. ${ }^{9}$ This method is simple and has a conservative methodology making it ideal for a set-up such as ours. We tried to study the effectiveness of this method with three years of treatment in children with idiopathic CTEV treated by the post graduates at our teaching hospital.

\section{METHODS}

In our prospective study total of 111 children with 166 club feet were treated using Ponseti method. ${ }^{10}$ Patients were selected from cases at our outpatient clinic at department of orthopaedics or those who were referred from department of paediatrics or OBGYN. Patients were treated by the post graduates/residents at our teaching hospital during the period of January 2012 and January 2017 with minimum follow up period of three years, after obtaining ethical clearance from the institutional review board.

All children with idiopathic club foot were included in the study. Secondary club foot, atypical club foot, cases with history of prior surgeries (complex club foot) and children with associated birth anomalies like neuromuscular problems, meningomyelocele, myelocele and arthrogryposis multiplex congenita were excluded from the study. All the children were clinically examined thoroughly including neuromuscular and neurological examination to rule out secondary causes and once confirmed to be idiopathic cause of CTEV, were included in the study. Significant and relevant data related to the patient were entered in a file specified to that patient and details were added or updated at every visit. Data file included details such as patient demographics, details of physical examination, age at which treatment was started, deformity assessment by Catterall-Pirani scoring system at initial visit as well as weekly follow up, number of weekly casts required before tenotomy, deformity correction details and type of cast applied, number of missed appointments, weather tenotomy was performed or not, tenotomy details and complications associated with it, pre and post tenotomy Pirani score, complications associated with casting, foot abduction splint details and patient compliance. ${ }^{11}$

Pirani score helps in assessing the deformity in foot and response to the treatment provided. It assesses the hind foot and mid foot deformities each of these have three components each. Hind foot components include posterior heel crease, emptiness of heel and rigidity of equinus. Mid foot components are curvature of lateral border of foot, medial crease and lateral head of talus coverage. Each component is given a score of one if fixed and present, half a point if mild and zero if absent. Maximum score of six represents severe club foot and minimum of zero implies a normal foot; corrected foot can sometimes have residual score of 0.5 or one with normal function.

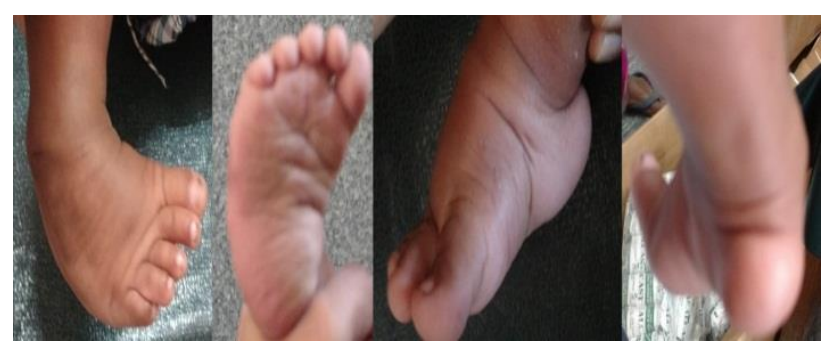

Figure 1: Abnormal foot at presentation showing varus deformity with forefoot adduction and deep medial and posterior crease.

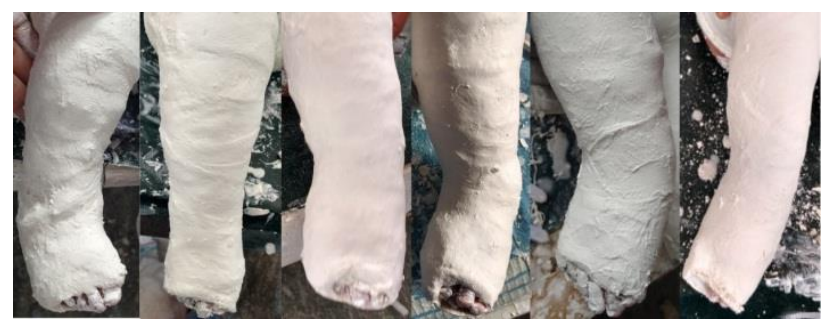

Figure 2: Serial casts showing improvement in abduction.

Treatment Regime: We used Ponseti method for correction of foot deformities (Figure 1) according to the following regime. Serial casts (Figure 2) were applied by gently manipulating the foot and followed up weekly for recasting noting Pirani score at every visit. The deformities were corrected in the following order of 
cavus, adduction, varus and equinus. Adduction and varus deformities were simultaneously corrected while casting. Cavus deformity was corrected by supinating the foot along with elevation of first metatarsal. Once the cavus was corrected adduction and varus deformities were corrected by carefully abducting the foot by giving counter pressure over the talar head taking care to never dorsiflex or pronate the foot. Technique of applying the cast was same for every patient with appropriate manipulation depending upon the stage of correction. The babies were breastfed before the procedure and mothers were made to hold their children so that they would be comfortable and not cry during the procedure. An assistant was made to hold the foot according to the correction required and three inch plaster of paris cast was applied after applying the soft band cotton roll. The casts were snuggly fitting making sure it was not too tight, cast was first applied below the knee to achieve appropriate position of foot and the converted to above the knee cast keeping knee in 90 degree of flexion and hips slightly externally rotated. Decreasing Pirani scores indicated that the treatment was working where as an increasing score suggested relapse or failure, in such cases casting was restarted from correction of cavus deformity again. Once we were able to achieve foot abduction of 70 degree but dorsiflexion of foot was less than 10 degrees percutaneous tenotomy (Figure 3) was performed. Before performing a tenotomy mid foot score had to be one or less with talar head coverage score of zero. All the tenotomies were performed under local aneathesia using a No. 15 blade by making a small puncture on the medial side over the insertion of the tendon, pressure was applied till hemostasis was achieved and cast was applied in a similar manner with foot in full abduction and dorsiflexion. This cast was left for three weeks and patients were the called for Steenbeek FAB (Figure 4) to help maintain the position and prevent relapse. The brace was adjusted to keep the affected foot in 70 degree abduction and maximum dorsiflexion, the size of the brace was checked to match the size of the feet and we made sure that the shoes fit appropriately. Parents were then advised about compliance with this device and additional follow-ups if there were issues with ill-fitting or loose shoes. Parents were asked to keep the splint on for no less than 23 hours a day for four months after tenotomy. After that, they were advised to apply the FAB only during day time naps and regular night naps up to the age of four. Patients were followed up on weekly basis initially then followed up once a month to check for compliance and later once in three months till the age of four.

Pirani score was monitored at every visit to check for relapse and final scores were assessed after minimum of three years of follow up. We categorized our final outcomes as excellent, good and poor based on final Pirani score. Excellent outcomes were graded for scores of zero, good when scores were 0.5 or 1 and poor if scores were more than one. To term treatment as successful we needed scores of less than one which implies good and excellent outcomes correspond to successful treatment (Figure 5). Poor outcomes where scores are more than one suggest treatment failure or relapse which requires reapplication of casts or other surgical intervention if necessary.

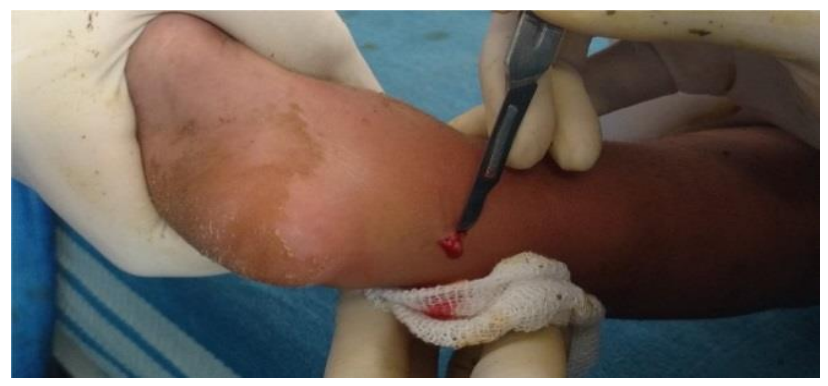

Figure 3: Percutaneous tenotomy.

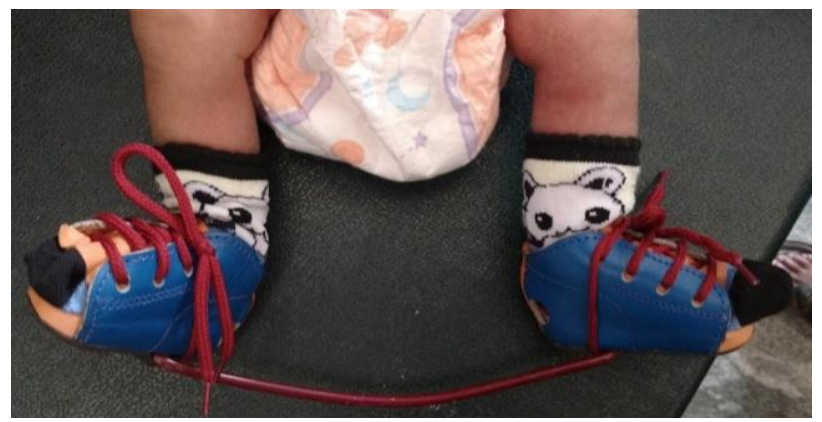

Figure 4: Steenbeek foot abduction brace.

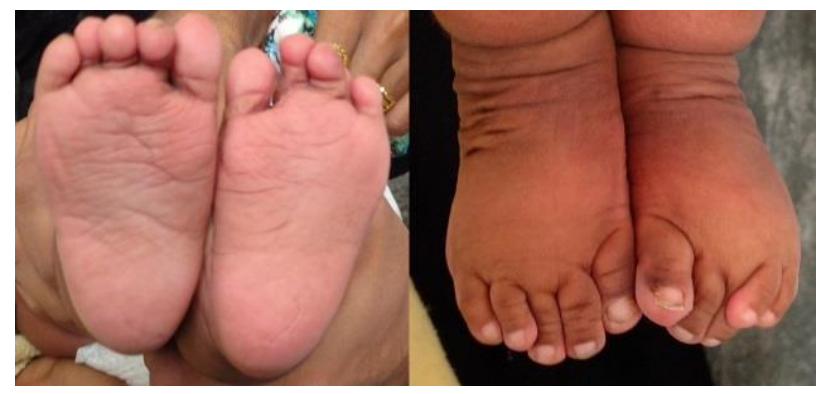

Figure 5: Corrected club foot (right side).

Descriptive statistics were used to compare Pirani scores at presentation and final visits.

\section{RESULTS}

Patient demographics are highlighted in Table 1.

Table 1: Patient demographics.

\begin{tabular}{|ll|}
\hline Number of patients : Feet & $\mathbf{1 1 1 : 1 6 6}$ \\
\hline Sex - Male : Female & $72: 39$ \\
\hline Involvement - Unilateral : Bilateral & $56: 55$ \\
\hline Side in unilateral - Right : Left & $31: 25$ \\
\hline Age - <1 month : >1 month & $61: 50$ \\
\hline Order of birth - First born : Later born & $72: 39$ \\
\hline
\end{tabular}


All the cases treated were of idiopathic club foot, other causes were excluded from the study. At the beginning of the treatment mean Pirani score was 5.5 (range 4-6). 120 $(72.29 \%)$ club feet had Pirani score of six and 46 $(27.71 \%)$ with scores of $<6$. On an average 5.7 casts (range 3-9) were required to achieve adequate score to perform tenotomy. $100 \%$ of the patients under went tenotomy. All the patients were given FAB four weeks after final cast post tenotomy.

Initial correction was obtained in all of 160 (96.39\%) feet within four months of follow up using the Ponseti method with Pirani score of one and below. Worsening of scores or relapse of deformity was seen in $6(3.61 \%)$ feet in three children. This worsening of score was mainly attributed to improper use of the FAB and noncompliance with the brace as recurrence was only noted in these three patients. Only four patients did not follow up and defaulted so, were excluded from the study.

Most common complication noted was cast related such as loose cast or cast breakage seen in nine patients, swelling of feet was seen in five children. Next most common complication was blister formation and skin excoriations seen in three children. There were no complications reported after tenotomy and there were no reported serious bleeding or wound issues at the tenotomy site.

Table 2: Final Pirani score and treatment outcome.

\begin{tabular}{|llll|}
\hline $\begin{array}{l}\text { Pirani } \\
\text { score }\end{array}$ & $\begin{array}{l}\text { Total number of } \\
\text { feet } \mathbf{n} \mathbf{1 6 6}(\boldsymbol{\%})\end{array}$ & $\begin{array}{l}\text { Treatment } \\
\text { outcome }\end{array}$ & $\begin{array}{l}\text { Final } \\
\text { result }\end{array}$ \\
\hline $\mathbf{0}$ & $97(58.43)$ & Excellent & Successful \\
\hline $\mathbf{0 . 5}$ & $40(24.10)$ & Good & Successful \\
\hline $\mathbf{1}$ & $23(13.86)$ & Good & Successful \\
\hline $\mathbf{> 1}$ & $6(3.61)$ & Poor & Failure \\
\hline
\end{tabular}

After three years of follow up we found that mean Pirani score was 0.26 in 160 club feet, six club feet were excluded as there was recurrence in these cases. We achieved excellent results outcomes in $97(58.43 \%)$ club feet with Pirani scores of zero after three years of follow up. Good results with scores between 0.5-1 were seen in $63(37.96 \%)$ feet. Poor outcomes with scores of $>1$ were seen only in three cases in six $(3.61 \%)$ feet, these cases required further surgical interventions. Which shows our treatment was successful in $96.39 \%$ of cases. Results have been summarized in Table 2 .

\section{DISCUSSION}

CTEV is a very common but complex problem in pediatric patients; it can lead to serious difficulties if neglected. Club feet have been managed by various modalities including surgical approach but Ponseti method using serial casts and manipulation with or without use of tenotomy have shown to be effective and convenient if followed up adequately. This method has shown to have very good long term success rate. ${ }^{10-12}$ Surgical options are invasive and are usually associated with stiffness and weakness which makes Ponseti technique a preferred method. ${ }^{13,14}$ Most of the studies show good short term results but there are very few studies with larger sample size and long term results. ${ }^{14-17}$ Various reasons for failure to correct the deformity include improper casting, inadequate tenotomy or under correction of deformity, improper use or application of splint and poor compliance of patients. ${ }^{18,19}$

In our study we had 6 feet with relapse all of them were due to improper use of the FAB where the patient was non- compliant with the instructions given and would not keep the brace on for adequate hours recommended. We tried to overcome this problem but having special sessions on regular basis to educate and make the parents aware about the problem and the importance of right usage of Steenbeek brace and plaster care, we also motivated the parents to not miss appointments to avoid any drop outs. All the patients with relapse or recurrence were restarted with casting for three to six weeks with further manipulations and tenotomy with proper usage of food abduction brace. If not corrected further surgical management was considered.

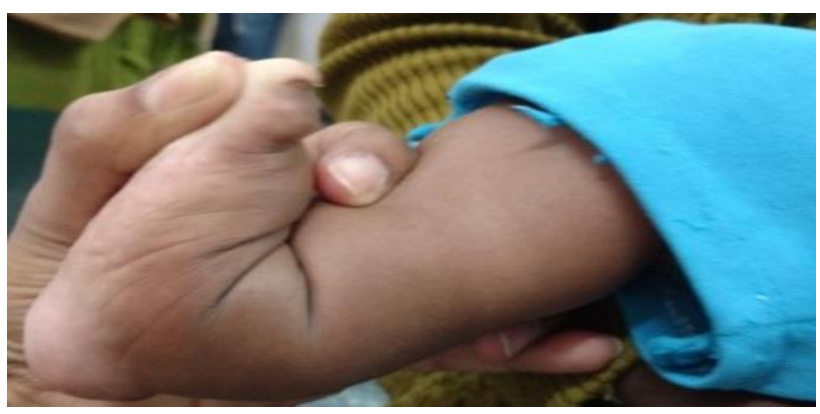

Figure 6: Good dorsiflexion achieved after tenotomy.

For a long time it was believed that children who presented early had better results with lower failure rates. ${ }^{14}$ But many studies now suggest that age of presentation does not affect the final outcome in terms of achieving good correction and plantigrade foot. ${ }^{19-21}$ Most children in our study were below the age of one month and majority below the age of one, few more than one year. We found no correlation between age of presentation and final outcome in these patients, although these patients required more number of weekly casts before tenotomy. Average number of overall casts per foot was 5.7 casts which is less when compared with average of 7.6 casts with most children requiring five to 10 casts during treatment as shown by Ponseti et al in their series. ${ }^{22,23}$ The number of casts required also depends on the experience and the technique of application and more experienced clinicians require fewer castes. ${ }^{24}$ We performed tenotomy on $100 \%$ of our cases. The reason why tenotomy rates are higher in our study is because of functional requirements of the patients which require squatting so this would allow us to achieve 
greater dorsiflexion of 10-15 degrees or more (Figure 6). Compared to our study $84.2 \%$ of cases required tenotomy in a study by Pavone et al whereas Bor et al documented $92.3 \%$ cases. $^{25,26}$ We did not encounter any serious complications after tenotomy, although swelling of feet, skin excoriations or pressure sore and cast associated complications were noted. Results of four studies are compared in Table 3.

Table 3: Results of Ponseti method compared in four studies.

\begin{tabular}{|c|c|c|c|c|}
\hline & Present study & Pavone et $\mathrm{al}^{25}$ & Bor et $\mathbf{a l}^{26}$ & Saif Ullah et al $^{29}$ \\
\hline Patients & 111 & 82 & 74 & 38 \\
\hline Number of club feet & 166 & 114 & 117 & 58 \\
\hline Male: Female & 1.85 & 2.1 & 1.8 & 2 \\
\hline Bilaterality & $55(49.6 \%)$ & $32(39.0 \%)$ & $43(58.1 \%)$ & $20(52.6 \%)$ \\
\hline Pirani score at presentation & 5.5 & 5.56 & 5.09 & 5.57 \\
\hline Number of casts & 5.7 & 6.6 & 6.3 & 3.75 \\
\hline Tenotomy & $166(100 \%)$ & $96(84.2 \%)$ & $108(92.3 \%)$ & $50(86.2 \%)$ \\
\hline Satisfactory outcome & $96.4 \%$ & $95.6 \%$ & $89.2 \%$ & $96.6 \%$ \\
\hline Failure/ Relapse & $6(3.6 \%)$ & $3(3.7 \%)$ & $24(32.4 \%)$ & $2(3.4 \%)$ \\
\hline
\end{tabular}

Changulani et al had reported neurovascular damage and Dobbs et al had reported serious bleeding due to peroneal artery and lesser saphenous vein injury. ${ }^{27,28}$ We were able to successfully correct $96.39 \%$ of CTEV feet showing excellent to good outcomes with Pirani score or $<1$ compared to $96.6 \%$ feet corrected by Ullah et al. ${ }^{29}$ Radiographic assessment was not done in our study as study by Roye et al shows that radiographic data had poor utility in evaluation of the outcome. ${ }^{30}$ Patients can have very good functional feet despite having imperfect feet radiological. ${ }^{31}$ This is the reason we used clinical evaluation including Pirani score to assess to outcome in our patients to give functional, plantigrade and acceptable feet.

\section{CONCLUSION}

Ponseti method of treatment for CTEV is very effective, simple, non-invasive and convenient with excellent outcomes over long term with no significant complications. This condition causes unnecessary physical, psychological, social and economic burden on patients and their families. This is why counselling and awareness among parents is important in terms of achieving effective results using Ponsti method, especially in developing countries. We were able to give children functional, flexible, painless, plantigrade and cosmetically acceptable feet without recurrence if parents were adherent with the protocol. Most important aspect of treatment success is dependent on patient compliance and right use of the FAB as instructed.

Ponseti cast can be applied by any one with minimal training to achieve good results as shown by post graduates in our study. Tenotomy should be performed in all cases to achieve good function and minimize recurrence.

\section{ACKNOWLEDGEMENTS}

We would like to thank our staff and professors from department of Orthopedics, Pediatrics and OBGYN for providing their insight and expertise which greatly assisted our research.

\section{Funding: No funding sources}

Conflict of interest: None declared

Ethical approval: All procedures performed in studies involving human participants were in accordance with the ethical standards of the institutional and/or national research committee and with the 1964 Helsinki declaration and its later amendments or comparable ethical standards

\section{REFERENCES}

1. Dobbs MB, Rudzki JR, Purcell DB, Walton T, Porter KR, Gurnett CA. Factors predictive of outcome after use of the Ponseti method for the treatment of idiopathic clubfeet. J Bone Joint Surg (Am). 2004;86(1):22-7.

2. Ponseti IV. Idiopathic congenital talipes equinovarus. In: Ponseti IV, eds. Congenital Clubfoot Fundamentals of Treatment. 1st ed. New York: Oxford University Press Inc. 1996;1(2):448-54.

3. Kite JH. Some suggestions on treatment of clubfoot by casts. J Bone Joint Surg Am. 1963;45:406-12.

4. Simons GW. Analytical radiography and the progressive approach in talipes equino varus Orthop Clin of North Am. 1978;9(1):187-206.

5. Cummings RJ, Lovell WW. Operative treatment of congenital idiopathic club foot. J Bone Joint Surg Am. 1988;70(7):1108-12.

6. Harrold AJ, Walker CJ. Treatment and prognosis in congenital clubfoot. Bone Joint Surg Br. 1983;65(1):8-11.

7. Yamamoto H, Muneta $\mathrm{T}$, Morita S. Non-surgical treatment of congenital clubfoot with manipulation cast and modified Denis-Browne Splint. Pediatr Orthop. 1998;18(4):538-42.

8. Cooper DM, Dietz FR. Treatment of idiopathic clubfoot. A thirty year follow-up note. J Bone Joint Surg. 1995;77(10):1477-89. 
9. Tindall AJ, Steinlechner CW, Lavy CB, Mannion S, Mkandawire N. Results of manipulation of idiopathic club deformity in Malawi by orthopaedic clinical officers using the Ponseti method: a realistic alternative for the developing world? J Pediatr Orthop. 2005;25(5):627-9.

10. Ponseti IV. Treatment of congenital clubfoot. J Bone Joint Surg Am. 1992;74(3):448-54.

11. Dyer PJ, Davis N. The role of the Pirani scoring system in the management of club foot by the Ponseti method. J Bone Joint Surg Br. 2006;88(8):1082-4.

12. Ponseti IV. The treatment of congenital clubfoot. J Orthop Sports Phys Ther. 1994;20(1):1.

13. Lehman WB, Mohaideen A, Madan S, Scher DM,Van Bosse HJ, Iannacone M, Bazzi JS, Feldman DS. A method for the early evaluation of the Ponseti (Iowa) technique for the treatment of idiopathic clubfoot. J Pediatr Orthop. 2003;12(2): 133-40.

14. Abdelgawad AA, Lehman WB, Van Bosse HJ, Scher DM, Sala DA. Treatment of idiopathic clubfoot using the Ponseti method: minimum 2-year followup. J Pediatr Orthop B. 2007;16(2):98-105.

15. Colburn M, Williams M. Evaluation of the treatment of Idiopathic clubfoot by using the Ponseti Method. J Foot Ankle Surg. 2003;42(5):259-67.

16. Cohen-Sobel E, Caselli M, Giorgini R, Stummer S. Long-term follow-up of clubfoot surgery: analysis of 44 patients. J Foot Ankle Surg. 1993;32(4):41123.

17. Laaveg SJ, Ponseti IV. Long-term results of treatment of congenital clubfoot. J Bone Joint Surg Am. 1980;62(1):23-31.

18. Ippolioto E, Farsetti P, Caterini R, Tudisco C. Longterm comparative results in patients with congenital clubfoot treated with two different protocols. J Bone Joint Surg Am. 2003;85(7):128694.

19. Morcuende JA, Dolan LA, Dietz FR, Ponseti IV. Radical reduction in the rate of extensive corrective surgery for clubfoot using the Ponseti method. Pediatrics. 2004;113(4):376-80.

20. Morcuende JA, Abbasi D, Dolan LA, Ponseti IV. Results of an accelerated Ponseti protocol for clubfoot. J Pediatr Orthop. 2005;25(5):623-6.
21. Lourenco AF, Morcuende JA. Correction of neglected idiopathic club foot by the Ponseti method. J Bone Joint Surg Br. 2007;89(3):378-81.

22. Ponseti IV. Clubfoot Management. Editorial. J Pediatric Orthopaedics. 2000;20(6):699-700.

23. Ponseti IV. Relapsing clubfoot: causes, prevention, and treatment. Iowa Orthop J. 2002;22:55-6.

24. Roye BD, Vitale MG, Gelijns AC, Roye DP Jr. Patient-based outcomes after clubfoot surgery. J Pediatr Orthop. 2001;21(1):42-9.

25. V. Pavone, G. Testa, L. Costarella, P. Pavone, G. Sessa. Congenital idiopathic talipes equinovarus: aneveluation in infants treated by the ponseti method. European review for medical and pharmacological sciences. 2013;17:2675-9.

26. Bor N, Coplan JA, Herzenberg JE. Ponseti treatment for idiopathic clubfoot: minimum 5-year follow up. Clin Orthop Relat Res. 2009;467(5):1263-70.

27. Changulani M, Garg NK, Rajagopal TS, Bass A, Nayagam SN, Sampath J, et al. Treatment of idiopathic club foot using the Ponseti method-Initial Experience. J Bone Joint Surg Br. 2006;88(10):1385-7.

28. Dobbs MB, Gordon JE, Walton T, Schoenecker PL. Bleeding complications following percutaneous tendoachilles tenotomy in the treatment of clubfoot deformity. J Pediatr Orthop.2004;24(4):353-7.

29. Md. Saif Ullah, Kazi Md. Noor-ul Ferdous, Md. Shahjahan, Sk. Abu Sayed. Management of Congenital Talipes Equino Varus (CTEV) by Ponseti Casting Technique in Neonates: Our Experience. J Neonatal Surg. 2013;2(2):17.

30. Roye BD, Vitale MG, Gelijns AC, Roye DP Jr. Patient-based outcomes after clubfoot surgery. J Pediatr Orthop. 2001;21(1):42-9.

31. Hutchins PM, Foster BK, Paterson DC, Cole EA. Long term results of early surgical release in club feet. J Bone Joint Surg Br. 1985;67(5):791-9.

Cite this article as: Bansal R, Jolly A, Farook PBM, Kamran I, Wahaj S, Hanasoge V. Management of congenital talipes equino varus using Ponseti method: 3 year follow up in 166 club feet. Int J Res Orthop 2017;3:775-80. 